\title{
QUEEN'S
UNIVERSITY
BELFAST
}

\section{The Expression of Terpenoid Indole Alkaloid (TIAs) Pathway Genes in Catharanthus roseus in Response to Salicylic Acid treatment}

\author{
Soltani, N., Nazarian Firouzabadi, F., Shafeinia, A., Sadr, A. S., \& Shirali, M. (2020). The Expression of \\ Terpenoid Indole Alkaloid (TIAs) Pathway Genes in Catharanthus roseus in Response to Salicylic Acid \\ treatment. Molecular Biology Reports. https://doi.org/10.1007/s11033-020-05759-y
}

Published in:

Molecular Biology Reports

Document Version:

Peer reviewed version

Queen's University Belfast - Research Portal:

Link to publication record in Queen's University Belfast Research Portal

Publisher rights

Copyright 2020 Springer. This work is made available online in accordance with the publisher's policies. Please refer to any applicable terms of use of the publisher.

\section{General rights}

Copyright for the publications made accessible via the Queen's University Belfast Research Portal is retained by the author(s) and / or other copyright owners and it is a condition of accessing these publications that users recognise and abide by the legal requirements associated with these rights.

Take down policy

The Research Portal is Queen's institutional repository that provides access to Queen's research output. Every effort has been made to ensure that content in the Research Portal does not infringe any person's rights, or applicable UK laws. If you discover content in the Research Portal that you believe breaches copyright or violates any law, please contact openaccess@qub.ac.uk. 
The Expression of Terpenoid Indole Alkaloid (TIAs) Pathway Genes in Catharanthus roseus in Response to

\section{Salicylic Acid treatment}

Narges Soltani ${ }^{1}$, Farhad Nazarian-Firouzabadi ${ }^{1}$, Alireza Shafeinia ${ }^{2}$, Ayeh Sadat Sadr ${ }^{3}$ and Masoud Shirali ${ }^{4,5}$

\section{Corresponding Author:}

Farhad Nazarian Firouzabadi: nazarian.f@lu.ac.ir

1. Production engineering and plant genetics department, Faculty of Agriculture, Lorestan University, P.O. Box 465, Khorramabad, Iran.

2. Production engineering and plant genetics department, Ramin Agriculture and Natural Resource University of Khuzestan, Iran.

3. Aquaculture Research Center-South of Iran, Iranian Fisheries Science Research Institute, Agricultural Research Education and Extension Organization (AREEO), Ahvaz, Iran.

4. Agri-Food and Biosciences Institute, Hillsborough BT26 6DR, UK.

5. School of Biological Sciences, Queen's University Belfast, Belfast, BT9 5AJ, UK

\section{Abbreviations}

AS: Anthranilate Synthase

CM: Chorismate Mutase

DAT: Deacetylvindoline-4-O-acetyltransferase

D4H: Desacetoxyvindoline-4-hydroxylase

G10H: Geraniol-10-hydroxylase

RSP9: 40S ribosomal protein S9

SA: Salicylic Acid

SLS: Secologanin Synthase

STR: Strictosidine Synthase

TDC: Tryptophan Decarboxylase

TIAs: Terpenoid Indole Alkaloids 


\begin{abstract}
Vinblastine and vincristine are two important anti-cancer drugs that are synthesized by the Terpenoid Indole Alkaloids (TIAs) pathway in periwinkle (Catharanthus roseus). The major challenge in the pharmaceutical industry is the low production rate of these alkaloids. TIAs pathway is flexible and is affected by elicitors, such as Salicylic Acid (SA). This study aimed to investigate the expression pattern of some key genes in TIAs pathway under SA treatment. Foliar application of SA $(0.01$ and $0.1 \mathrm{mM})$ was used and leaves samples were taken at $0,12,18,24$ and 48 hours after the treatment. qRT-PCR was used to investigate the expression pattern of Chorismate mutase $(\mathrm{Cm})$, Tryptophan decarboxylase (Tdc), Geraniol-10-hydroxylase (G10h), Secologanin synthase (Sls), Strictosidine synthase (Str), Desacetoxyvindoline-4-hydroxylase (D4h) and Deacetylvindoline-4-O-acetyltransferase (Dat) genes, following the SA treatment. The results of this experiment showed that transcript levels of Tdc, G10h, Sls, Str, D4h and Dat genes were significantly up-regulated in both SA concentration treatments. Furthermore, the highest transcript levels of Dat was observed after 48 hours of the SA treatments. qRT-PCR results suggests that SA induces transcription of major genes involved in alkaloids biosynthesis in Catharanthus roseus. It can be concluded that up-regulation of Tdc, G10h, Sls, Str, D4h and Dat genes can result in a higher production rate of vinblastine and vincristine alkaloids.
\end{abstract}

\title{
Keywords
}

Elicitor, Gene Expression, Salicylic Acid, Terpenoid Indole Alkaloids, TIAs pathway, qRT-PCR

\section{Introduction}

Madagascar Periwinkle (Catharanthus roseus), a member of Apocynaceae family, is an important medicinal plant native to Madagascar [1,2]. It is also known as Cape periwinkle, Vinca rosea, Rosy periwinkle, Rose periwinkle, Ammocallis rosea, and Lochnera rosea [3]. C. roseus is an excellent model system plant to study the plants' secondary metabolome [4] due to its small genome size (1500 Mbp), herbaceous perennial habit, short life cycle, and sexual and vegetative modes of propagation, [5,6,4]. Periwinkle is rich in alkaloids, phenols, steroids, anthocyanins, saponins, and carbohydrate compounds [1,5], with over 400 alkaloids [2] and more than 130 Terpenoid Indole Alkaloids (TIAs) [7-9]. Periwinkle possesses anti-oxidant, anti-bacterial, anti-ulcer, anti-diarrheal activities, and it is used to treat cancer, diabetes, blood pressure, asthma, mouth ulcers, and constipation [1,3]. Vincristine and vinblastine, as two important alkaloids used in the treatment of cancer [3,8] are solely synthesized by the TIAs pathway in the leaves and 
stems of the C. roseus $[1,3,8]$. Despite the huge interest and demand by the pharmaceutical industry regarding the production of these vital alkaloids, they are naturally produced in a very low amount in the C. roseus $[10,11,8,12]$. Furthermore, their laboratory synthesis by the chemical methods faces some obstacles due to their complex chemical structures $[13,8]$. The TIAs biosynthesis pathway leading to the production of pharmaceutical valuable compounds seems to be highly complex involving at least 35 intermediates and 30 enzymes from indole pathway, methylerythritol 4-phosphate (ME), terpenoid, and alkaloid pathways [9].

Tryptamine is produced from the tryptophan through the activity of tryptophan decarboxylase (TDC) [14] and the indole pathway that consequently serves as the indole moiety for production of the TIAs $[15,16,8]$. Secologanin synthase (SLS) is a member of P450 enzymes that catalyzes an unusual ring-opening reaction of loganin leading to the secologanin production [8]. In the alkaloids pathway, tabersonine is converted into different compounds, especially vindoline, a chemical precursor to vinblastine by deacetylvindoline-4-O-acetyltransferase (DAT) [13]. Finally, vinblastine and vincristine are synthesized by the compression of catharanthine and vindoline metabolites [11]. Production of diverse types of TIAs pathway metabolites is controlled at transcriptional, translational, and posttranslational levels [8] and is fluctuated by many internal and external elicitors. For instance, phytohormones including Salicylic Acid (SA) and Ethylene (ET) stimulate the production rate of catharanthine, vindoline, and vinblastine in the C. roseus, whereas Abscisic Acid (ABA) and Gibberellic Acid (GA) tend to decrease the production rate of TIAs [13]. However, the signal transduction pathway mechanisms explaining how these growth hormones regulate TIAs biosynthesis are largely unknown. Many factors such as SA have been reported to regulate TIAs pathway $[17,8]$. SA is involved in many physiological processes such as plant growth and development, flowering, ion uptake, stomatal closure, disease resistance, and heat production [18,19]. SA, as a plant signaling molecule improves the anti-oxidant defense system of the C. roseus, and plays a pivotal role in combating the abiotic stresses such as drought, salinity, cold and restraining nickel toxicity as well as increasing the amount of vincristine and vinblastine alkaloids $[13,20,21,17,11]$. For instance, Pan et al. [13] have shown that spraying the SA at $0.1 \mathrm{mM}$ concentration on the $C$. roseus leaves significantly increased the accumulation of catharanthine, vinblastine, and vindoline alkaloids. It has also been reported that spraying $0.01 \mathrm{mM}$ of SA under water [22] and salinity [20] stresses reduces the harmful effects of abiotic stresses eventually leading to an increase in the total amount of alkaloids, vincristine, and vinblastine in the C. roseus. Despite SA benefits, the negative effects of surplus application of SA have also been reported and its efficiency depends on the age of plant and concentration of SA [18]. 
In the present study, the expression level seven major genes associated with indole, terpenoid and alkaloids pathways was evaluated by the quantitative Real Time Polymerase Chain Reaction (qRT-PCR) analysis after application of the SA (0.01 and $0.1 \mathrm{mM})$.

\section{Materials and Methods}

\section{Plant Material and Treatments}

The Periwinkle seeds were obtained from Pan American Seed Company (USA) and were cultivated in the pots (10 $\mathrm{cm}$ diameter $\times 10 \mathrm{~cm}$ height $)$ and then, were maintained in a growth chamber $\left(25^{\circ} \mathrm{C}, 16 \mathrm{~h}\right.$ photoperiod, and light intensity of $70 \% \mu \mathrm{mol} \mathrm{m}^{-2} \mathrm{~s}^{-1}$ ). SA was sprayed at concentrations of 0.01 and $0.1 \mathrm{mM} \mathrm{[23]} \mathrm{at} \mathrm{the} \mathrm{beginning} \mathrm{of} \mathrm{flowering}$ stage [24,5]. Samples were taken from the third and fourth leaves as described by Roepke et al. [25]. The sampling was carried out at $12,18,24$, and 48 hours after the treatment ( 3 replicates each), and the samples were immediately frozen in the liquid nitrogen and were stored at $-80^{\circ} \mathrm{C}$ for RNA extraction.

\section{RNA Extraction, cDNA Synthesis, and Primer Designing}

Total RNA was extracted from $100 \mathrm{mg}$ of leaves using the Monarch $^{\circledR}$ Total RNA Miniprep Kit (NEB Company, UK. Cat\# T2010) according to the manufacturer's instructions. RNA concentration was quantified using the NanoDrop 2000c Spectrophotometer (Thermo Scientific NanoDrop 2000, USA) and were qualified by $1 \%$ agarose gel electrophoresis. The first strand cDNA was synthesized from $500 \mathrm{ng}$ of total RNA using the Moloney-Murine Leukemia Virus (M-MLV) reverse transcriptase according to the manufacturer's instructions in the final volume of $20 \mu \mathrm{L}$ (RNase $\mathrm{H}^{-}$) Kit (NEB Company, UK. Cat\# E6300S). The cDNA was diluted to $100 \mathrm{ng} / \mu \mathrm{L}$ as the template for the real-time PCR analysis.

The sequences of target genes (Cm, Tdc, G10h, Sls, Str, D4h and Dat) and the 40S ribosomal protein S9 (Rsp9) reference gene were downloaded from the Genbank database. Primers were designed (Table 1) by the Oligo7 and Primer3Plus softwares except $\mathrm{Cm}$ primers, which had been previously designed and validated by Sun et al., (2016).

\section{Gene Expression Profiling and Analysis}

The qRT-PCR analysis (Step One Plus Real-time PCR System, Applied Biosystems, USA) was performed using the specific primers to ensure amplification of the target genes. The expression level of the target and reference genes was 
determined using the SYBR Green PCR Master Mix (NEB Company, UK. Cat\# M3003S). Each reaction was consisted of a mixture containing $16 \mathrm{ng} / \mu \mathrm{L}$ of cDNA ( $2 \mu \mathrm{L}$ of initial concentration in $100 \mathrm{ng} / \mu \mathrm{L}), 0.4 \mu \mathrm{M}$ of each of the forward and reverse primers, and SYBR Green PCR master mix (1X). The qRT-PCR was performed in three biological and three technical replicates. The thermal cycling conditions included an initial denaturation step at $95^{\circ} \mathrm{C}$ for $10 \mathrm{~min}$, followed by 40 cycles at $95^{\circ} \mathrm{C}$ for $30 \mathrm{~s}, 60^{\circ} \mathrm{C}$ for $30 \mathrm{~s}$, and $72^{\circ} \mathrm{C}$ for $30 \mathrm{~s}$. After each cycle, melting curve analysis was conducted by increasing the temperature from 60 to $95^{\circ} \mathrm{C}$ along with continuous monitoring of the SYBR Green fluorescence. The relative gene transcription was quantified using the comparative threshold cycle (CT) method and the data were analyzed using the REST ${ }^{\circledR}$ software [26] according to the $\Delta \Delta \mathrm{CT}$ method [27].

\section{Results}

The elicitor effect of SA was investigated on gene expression of the TIAs pathway. Since $\mathrm{Cm}$ and Anthranilate synthase (As) genes compete for the same precursor (Chorismate); the expression pattern of $\mathrm{Cm}$ was monitored at two different concentrations of SA. Although, the transcript level of the $\mathrm{Cm}$ gene showed a down-regulation at $\mathrm{C} 2(0.01$ mM of SA) and up-regulation at $\mathrm{C} 1(0.1 \mathrm{mM}$ of SA) (Fig. 1A), the higher amount of the SA led to significant upregulation of $\mathrm{Cm}$ only at $24 \mathrm{~h}$ after the treatment. No significant $(\mathrm{P}>0.5)$ differences were found between different time points after the SA application at lower concentrations.

In the indole pathway, the mRNA transcripts of the $T d c$ gene were elevated following the application of both concentrations of SA (Fig. 1B). The expression of the Tdc gene was up-regulated at C2 concentration, and the transcription level was slowly increased from $12 \mathrm{~h}$ to $24 \mathrm{~h}$. The highest level of transcription was observed at $48 \mathrm{~h}$. Similarly, the mRNA transcript level was up-regulated at $\mathrm{C} 1$ concentration, and the highest level was observed at $48 \mathrm{~h}$. The transcript level showed a significant up-regulation trend in both treatments except at $24 \mathrm{~h}$ at $\mathrm{C} 1$ concentration and reached a peak value at $48 \mathrm{~h}$ ( 3.76 and 3.82 fold changes, respectively).

The transcript mRNA level of the G10h gene in the terpenoid pathway was significantly up-regulated at 24 and $48 \mathrm{~h}$ following the application of the $\mathrm{C} 1$ concentration of SA (Fig. 2A). The expression of the G10h gene was slowly increased, and the highest mRNA transcript level was observed at 48h. Interestingly, the mRNA transcript level of G10h gene was significantly increased during $12 \mathrm{~h}-24 \mathrm{~h}$. The expression of the G10h gene was increased by 2.82 and 7.27 folds at $48 \mathrm{~h}$, respectively. 
The Sls mRNA transcript level was significantly increased over time with the highest level observed at $48 \mathrm{~h}$ post SA treatment at $\mathrm{C} 1$ concentration (Fig. 2B), which was similar to that of the G10h gene in terms of changes over time points and at various SA concentrations. The expression of Sls gene was significantly increased at C2 concentration, at 12 and $48 \mathrm{~h}$ but it was decreased at $24 \mathrm{~h}$.

Strictosidine synthase (Str) activity play a major role at the beginning of the alkaloid pathway. The mRNA transcripts level of Str gene was increased after SA treatment (Fig 3 A). The expression of Str was significantly increased by time and reached the highest level 48 hours after SA treatment. The same trend was observed for desacetoxyvindoline-4hydroxylase (D4h) gene (Fig 3B). However, the expression level of D4h was significantly lower than Str gene.

The expression of the Dat gene showed a significant up-regulati

on following both treatments over time except at C2 concentration after 18h (Fig. 3C). However, increase in the transcription level at $18 \mathrm{~h}$ was lower than expression level at $12 \mathrm{~h}$. The highest expression was observed at both concentrations at $48 \mathrm{~h}$ and reached 7.98 and 8.2 fold changes at $\mathrm{C} 1$ and $\mathrm{C} 2$ concentrations, respectively.

\section{Discussion}

Plant Growth Regulators (PGRs) significantly affect the synthesis of secondary metabolites [23]. The mechanism of stress defense in plants is mediated through various signaling pathways and many defensive proteins and non-protein compounds. Abscisic Acid, Jasmonic Acid, Ethylene and Salicylic Acid (SA) involved in plant defense are important components of different signaling pathways. SA also play a major role as an antioxidative compound in plants. Besides association in cross-talking with other pathways mediating plant resistance, SA regulates the components of its own signaling pathway. It also plays an important role in increasing metabolic rates, which contributes to the plant's energysaving through alternative pathways accompanied by a change in the level of amino acids within the plant (War et al., 2011; Hassoon and Abduljabbar, 2019). For instance, the effect of SA on the alkaloid accumulation and enhancement of the plant defense system in $C$. roseus has been documented $[13,22,20,21,23]$. It has been shown that foliar application of SA at two concentrations $(0.01 \mathrm{mM}$ and $0.1 \mathrm{mM})$ significantly increased the amount of catharanthine, vindoline, vinblastine, and vincristine alkaloids in the C. roseus [23]. Since many alkaloids in the C. roseus are synthesized in the TIAs pathway, it has been shown that the changes in the transcription level of upstream genes affect the expression of downstream genes and consequently the synthesis of downstream alkaloids $[28,8]$. 
As and $\mathrm{Cm}$, the first genes involved in the indole and flavonoid pathways, respectively, compete for the same substrate, the chorismate precursor. As a result of such a competition, the activities of As and Cm enzymes influence the final product as well as the number of intermediate compounds in both pathways $[29,8]$. If a higher amount of chorismate enters the flavonoid and phenylpropanoid pathways, the reaction shifts to the synthesis of phenylalanine, tyrosine, flavonoids, and phenylpropanoids instead and production of indole terpenoid alkaloids [29]. Higher activity of As in the TIAs pathway leads to the fluctuation of downstream genes and downstream alkaloids $[29,15,8]$. Overexpression of the As gene in the transgenic C. roseus hairy root lines has been reported to be driven by an inducible promoter [8]. In line with these findings, in the present study, the foliar application of SA did not show a constant trend (Fig. 1A). Tdc gene encodes the final enzyme in the TIAs indole pathway and converts tryptophan to tryptamine. Later, tryptamine enters the TIAs alkaloid pathway through the activity of strictosidine synthase (STR) enzyme [15,11]. Tdc and As genes are both active in the indole pathway. Overexpression of the As gene in the hairy roots of $C$. roseus has been found to result in the induction of As gene expression and subsequently the transcription level of the $T d c$ gene. The highest $T d c$ gene transcription rate was observed at $12 \mathrm{~h}$ after induction of As expression [8]. Results of a study on the Tdc gene transcription level and vindoline content following the treatment with the two fungal endophytes (Curvularia sp. CATDLF5 and Choanephora infundibulifera CATDLF6) in the C. roseus showed that the symbiosis significantly increased the transcript levels of the Tdc, As, G10h, Str, D4h, and Dat genes as well as the alkaloid content of vindoline [30]. Interestingly, in another study, the mRNA transcript level of $T d c$ was increased in the $C$. roseus seedlings following treatment with methyl jasmonate (MeJA). MeJA treatment led to an increase in the expression level of $T d c$ over time and the highest expression was observed at $0.2 \mathrm{mM}$ and $2 \mathrm{mM}$ concentrations after 48h [31]. Changes in the transcript levels of any of the upstream genes can also lead to changes in the transcript levels of the downstream genes depending on the position of genes in the TIAs cascade pathway. An increase in the Tdc transcript level and other TIAs indole pathway genes has been found to increase the production of the alkaloids [8,30,32]. In this study, the expression level of $T d c$ gene was up-regulated following the SA foliar application regardless of the SA concentration used. Despite the differences between the treatments applied in the present study and reported studies, an increase in the $T d c$ gene transcription level over time can lead to the induction of downstream genes in the TIAs cascade pathway and consequently accumulation of the alkaloids.

In this study, the level of mRNA transcripts of G10h and Sls genes belonging to the terpenoid pathway was investigated. G10H converts geraniol into 10-hydroxygeraniol, and SLS enzyme finally converts 10-hydroxygeraniol 
into secologanin. Later, secologanin enters the alkaloid pathway through the activity of the STR enzyme $[15,8,6]$. Studies have shown that the G10h gene plays an important role in the TIAs pathway [33] and any fluctuation in the G10h gene transcript level influences the alkaloid pathway and consequently the synthesis of commercial C. roseus alkaloids. In this regard, overexpression of the G10h and ORCA3 (Octadecanoid-derivative Responsive Catharanthus AP2-domain transcription factor) genes has been shown to increase the transcript level of the genes involved in the TIAs pathway including G10h, Tdc, As, Str, and D4h as well as an increase in the accumulation of strictosidine, vindoline, catharanthine, ajmalicine, and vinblastine alkaloids in the transgenic plants [28]. Furthermore, Papon et al., have shown that the combination of Cytokinin and Ethylene increased the accumulation of alkaloids and expression of G10h and MEP pathway genes but did not affect Tdc and Mevalonate (MVA) pathway genes [33]. In line with other reports [31,30], herein, an increase was observed in the G10h gene expression following SA application, suggesting that up-regulation of the genes involved in terpenoid pathway induces the transcription of associated genes and as a result an increase in the TIAs pathway end products $[28,8,30]$.

SLS is the final enzyme in the TIAs terpenoid pathway and converts loganin to secologenin. Secologanin and tryptamine enter the TIAs alkaloid pathway through the activity of the STR enzyme [15,32,34]. Report shows that the transcript levels of the Sls did not change during the treatment with symbiotic fungal endophytes [30]. A significant increase was found in the transcript level of the Sls mRNA gene in the C. roseus cell cultures after treatment with Cyclodextrins alone and combined co-incubation with MeJA and Cyclodextrins. Similar to the G10h gene, the expression of the Sls gene was elevated after treatment with MeJA and Cyclodextrins leading to a significant increase in the amount of ajmalicine and catharanthine alkaloids [32]. Although, our results were consistent with the findings of the studies by Almagro et al., (2014) and Pandey et al., (2016), no significant changes were found in the transcript level of the Sls gene after inoculation with two symbiotic fungal endophytes.

Tryptophan and secologanin are converted to the strictosidine by enzymatic activity of strictosidine synthase [30]. Change in expression level of Str gene can have a significant impact on expression of downstream genes in TIAs pathway [8]. Strictosidine, the product of strictosidine synthase activity, is a precursor important major genes of the alkaloid pathway, such as D4h, Dat, and $\operatorname{Prx} 1[6,15]$. Desacetoxyvindoline-4-hydroxylase (D4h) gene is one of the final genes in the alkaloid pathway that turns desacetoxyvindoline into deacetylvindoline and this product is one of the main precursors for making vindoline $[15,30]$. There is a direct link between an increase in vincristine accumulation and expression level of Dat and D4h genes $[11,30]$. DAT converts deacetylvindoline into vindoline in 
the TIAs alkaloid pathway $[15,6,8]$. An increase in the expression level of Dat has been found to result in the accumulation of vinblastine and vincristine alkaloids [12,35]. Inoculation of $C$. roseus with fungal endophytes led to an increase in the expression of Str, D4h and Dat genes and consequently vindoline content C. roseus increased [30]. Consistent with these findings, an increase has been reported in the accumulation of vindoline in the transgenic Datexpressing plants [12]. Furthermore, MeJA and putrescine treatments have been shown to lead to the up-regulation of Str, $\operatorname{Pr} x 1$, Dat genes and consequently the production of vincristine and vinblastine alkaloids [35]. In another study, foliar application of SA on the leaves of C. roseus seedlings exposed to drought and salinity stresses increased the transcript level of the Dat and D4h genes [11].

\section{Conclusion}

The results of this study showed a change in the expression of some TIAs pathway genes following the foliar application of SA. It is expected that the changes in the expression of the upstream genes will consequently influence the mRNA transcript level of the downstream genes and production of the final products. Foliar application of SA upregulated the transcript level of G10h and Sls genes. The treatments also increased the expression level of the Dat gene, which synthesizes the vindoline. It can be concluded that up-regulation of TIAs pathway genes can result in a higher production rate of vinblastine and vincristine alkaloids in the C. roseus.

\section{Compliance with ethical standards}

Conflict of interest Authors declare no conflicts of interest.

Ethical approval This article does not contain any studies with human participants or animals performed by any of the authors.

\section{References}


1. Sain M, Sharma V (2013) Catharanthus roseus (An anti-cancerous drug yielding plant). A Review of Potential Therapeutic Properties. Int J Pure App Biosci 1 (6):139-142

2. Lawal OA, Ogunwande IA, Ibirogba AE, Layode OM, Opoku AR (2015) Chemical Constituents of Essential Oils from Catharanthus roseus (L.) G. Don Grown in Nigeria. Journal of Essential Oil Bearing Plants 18 (1):57-63

3. Sandeep P, Jagjit K, Raman K, Kuldeep K (2014) Catharanthus roseus: A medicinal plant with potent anti-tumor properties. IntjResAyurveda Pharm 5 (6):5

4. Pandey-Rai S, Mallavarapu GR, Naqvi A, Yadav A, Rai SK, Srivastava S, Singh D, Mishra R, Kumar S (2006) Volatile components of leaves and flowers of periwinkle Catharanthus roseus (L.) G. Don from New Delhi. Flavour and fragrance journal 21 (3):427-430

5. Nejat N, Valdiani A, Cahill D, Tan Y-H, Maziah M, Abiri R (2015) Ornamental exterior versus therapeutic interior of Madagascar Periwinkle (Catharanthus roseus): the two faces of a versatile herb. The Scientific World Journal:20

6. Liu J, Cai J, Wang R, Yang S (2016) Transcriptional Regulation and Transport of Terpenoid Indole Alkaloid in Catharanthus roseus: Exploration of New Research Directions. International journal of molecular sciences 18 (1):53

7. Schluttenhofer C, Pattanaik S, Patra B, Yuan L (2014) Analyses of Catharanthus roseus and Arabidopsis thaliana WRKY transcription factors reveal involvement in jasmonate signaling. BMC genomics 15 (1):502

8. Sun J, Manmathan H, Sun C, Peebles CA (2016) Examining the transcriptional response of overexpressing anthranilate synthase in the hairy roots of an important medicinal plant Catharanthus roseus by RNA-seq. BMC plant biology 16 (1):108

9. Sun J (2016) Metabolic engineering and elucidation of the terpenoid indole alkaloid pathway in Catharanthus roseus hairy roots. Colorado State University. Libraries,

10. Carqueijeiro I, Noronha H, Duarte P, Gerós H, Sottomayor M (2013) Vacuolar transport of the medicinal alkaloids from Catharanthus roseus is mediated by a proton-driven antiport. Plant physiology $162(3): 1486-1496$

11. Mokhaberi A, Ahmadi J, Mafakheri S (2015) The expression profile of D4H and DAT genes in Catharanthus roseus in response to drought, salinity and salicylic acid. Iranian Journal of Genetics and Plant Breeding 2 (2):38-46

12. Wang Q, Xing S, Pan Q, Yuan F, Zhao J, Tian Y, Chen Y, Wang G, Tang K (2012) Development of efficient Catharanthus roseus regeneration and transformation system using Agrobacterium tumefaciens and hypocotyls as explants. BMC biotechnology 12 (1):34

13. Pan Q, Chen Y, Wang Q, Yuan F, Xing S, Tian Y, Zhao J, Sun X, Tang K (2010) Effect of plant growth regulators on the biosynthesis of vinblastine, vindoline and catharanthine in Catharanthus roseus. Plant growth regulation 60 (2):133-141

14. De Luca V, Marineau C, Brisson N (1989) Molecular cloning and analysis of cDNA encoding a plant tryptophan decarboxylase: comparison with animal dopa decarboxylases. Proceedings of the National Academy of Sciences 86 (8):2582-2586

15. Shukla AK, Khanuja SP (2013) Catharanthus roseus: The metabolome that represents a unique reservoir of medicinally important alkaloids under precise genomic regulation. In: OMICS Applications in Crop Science. CRC Press, pp 345-404

16. Almagro L, Fernández-Pérez F, Pedreño M (2015) Indole alkaloids from Catharanthus roseus: bioproduction and their effect on human health. Molecules 20 (2):2973-3000

17. Zhu J, Wang M, Wen W, Yu R (2015) Biosynthesis and regulation of terpenoid indole alkaloids in Catharanthus roseus. Pharmacognosy reviews 9 (17):24

18. Khandan-Mirkohi A, Khalili Halbi M, Salami SA, Lesani H (2017) Improving Effects of Mild Cold Stress and Salicylic acid on Growth and Physiology of Periwinkle (Catharanthus roseus Don.). International Journal of Horticultural Science and Technology 4 (1):67-78

19. Mustafa NR (2007) Retrobiosynthetic study of salicylic acid in Catharanthus roseus cell suspension cultures. Department of Pharmacognosy, Section Metabolomics, Institute of Biology ..., 
20. Idrees M, Naeem M, Aftab T, Khan MMA (2011) Salicylic acid mitigates salinity stress by improving antioxidant defence system and enhances vincristine and vinblastine alkaloids production in periwinkle [Catharanthus roseus (L.) G. Don]. Acta Physiologiae Plantarum 33 (3):987-999

21. Idrees M, Naeem M, Aftab T, Khan MMA (2013) Salicylic acid restrains nickel toxicity, improves antioxidant defence system and enhances the production of anticancer alkaloids in Catharanthus roseus (L.). Journal of hazardous materials 252:367-374

22. Idrees M, Khan MMA, Naeem M, Aftab T, Hashmi N, Alam M(2011) Modulation of defence responses by improving photosynthetic activity, antioxidative metabolism, and vincristine and vinblastine accumulation in Catharanthus roseus (L.) G. Don through salicylic acid under water stress. Russian agricultural sciences 37 (6):474-482

23. Kaur J, Singh A, Pathak T, Kumar K (2017) Role of PGRs in Anticancer Alkaloids (Vincristine and Vinblastine) Production. In: Catharanthus roseus. Springer, pp 309-319

24. Idrees M, Naeem M, KHAN MMA (2010) The superiority of cv 'rosea'over cv 'alba'of periwinkle (Catharanthus roseus L.) in alkaloid production and other physiological attributes. Turkish Journal of Biology 34 (1):81-88

25. Roepke J, Salim V, Wu M, Thamm AM, Murata J, Ploss K, Boland W, De Luca V (2010) Vinca drug components accumulate exclusively in leaf exudates of Madagascar periwinkle. Proceedings of the National Academy of Sciences 107 (34):15287-15292

26. Pfaffl MW, Horgan GW, Dempfle L (2002) Relative expression software tool (RESTC) for group-wise comparison and statistical analysis of relative expression results in real-time PCR. Nucleic acids research 30 (9):e36-e36

27. Livak KJ, Schmittgen TD (2001) Analysis of relative gene expression data using real-time quantitative PCR and the 2- $\Delta \Delta C T$ method. methods 25 (4):402-408

28. Pan Q, Wang Q, Yuan F, Xing S, Zhao J, Choi YH, Verpoorte R, Tian Y, Wang G, Tang K (2012) Overexpression of ORCA3 and $\mathrm{G} 10 \mathrm{H}$ in Catharanthus roseus plants regulated alkaloid biosynthesis and metabolism revealed by NMR-metabolomics. PloS one 7 (8):e43038

29. Mustafa NR, Verpoorte R (2007) Phenolic compounds in Catharanthus roseus. Phytochemistry Reviews 6 (2-3):243-258

30. Pandey SS, Singh S, Babu CV, Shanker K, Srivastava N, Shukla AK, Kalra A (2016) Fungal endophytes of Catharanthus roseus enhance vindoline content by modulating structural and regulatory genes related to terpenoid indole alkaloid biosynthesis. Scientific reports 6:26583

31. Wei S (2010) Methyl jasmonic acid induced expression pattern of terpenoid indole alkaloid pathway genes in Catharanthus roseus seedlings. Plant growth regulation 61 (3):243-251

32. Almagro L, Gutierrez J, Pedreño MA, Sottomayor M (2014) Synergistic and additive influence of cyclodextrins and methyl jasmonate on the expression of the terpenoid indole alkaloid pathway genes and metabolites in Catharanthus roseus cell cultures. Plant Cell, Tissue and Organ Culture (PCTOC) 119 (3):543-551

33. Papon N, Bremer J, Vansiri A, Andreu F, Rideau M, Crèche J (2005) Cytokinin and ethylene control indole alkaloid production at the level of the MEP/terpenoid pathway in Catharanthus roseus suspension cells. Planta medica 71 (06):572-574

34. de Bernonville TD, Foureau E, Parage C, Lanoue A, Clastre M, Londono MA, Oudin A, Houillé B, Papon N, Besseau S (2015) Characterization of a second secologanin synthase isoform producing both secologanin and secoxyloganin allows enhanced de novo assembly of a Catharanthus roseus transcriptome. BMC genomics 16 (1):619

35. Khataee E, Karimi F, Razavi K (2019) Alkaloids production and antioxidant properties in Catharanthus roseus (L.) G. Don. shoots and study of alkaloid biosynthesis-related gene expression levels in response to methyl jasmonate and putrescine treatments as eco-friendly elicitors. Biologia Futura 70 (1):38-46

\section{Legend to the Figures}


Figure 1. mRNA transcript fold changes of $\mathrm{Cm}(\mathrm{A})$, and $\mathrm{Tdc}(\mathrm{B})$ genes following treatment of Catharanthus roseus leaves with salicylic acid at two different concentrations $\left(\mathrm{C}_{1}: 0.1 \mathrm{mM}\right.$ and $\left.\mathrm{C} 2: 0.01 \mathrm{mM}\right)$ at 12, 18, 24 and 48 hours post treatment.

Figure 2. qRT-PCR expression analysis of G10h (A), and Sls (B) genes following treatment of Catharanthus roseus leaves with salicylic acid at two different concentrations $\left(\mathrm{C}_{1}: 0.1 \mathrm{mM}\right.$ and $\left.\mathrm{C} 2: 0.01 \mathrm{mM}\right)$ at 12, 18, 24 and 48 hours post treatment.

Figure 3. qRT-PCR expression analysis of Str (A), D4h (B) and Dat (C) after treatment of Catharanthus roseus leaves with salicylic acid at two different concentrations $(\mathrm{C} 1: 0.1 \mathrm{mM}$ and $\mathrm{C} 2: 0.01 \mathrm{mM})$ at 12, 18, 24 and 48 hours post treatment. 